
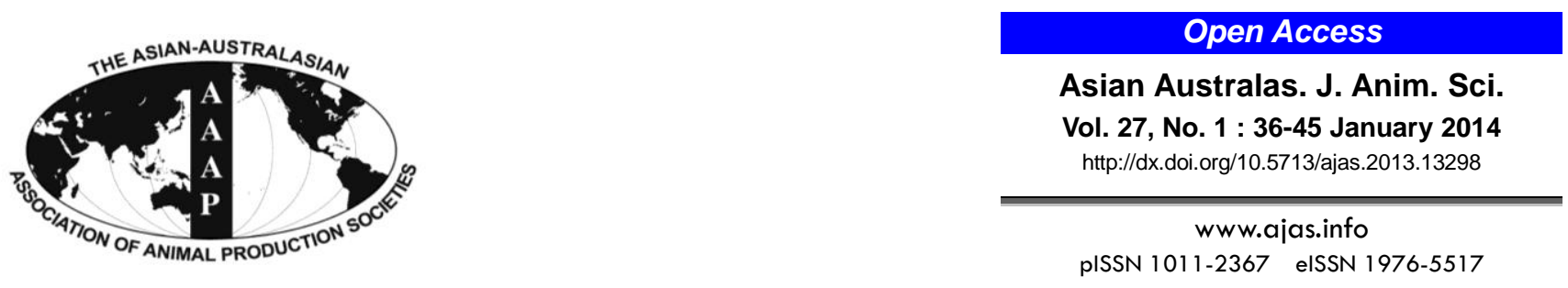

\title{
Influence of Yeast Fermented Cassava Chip Protein (YEFECAP) and Roughage to Concentrate Ratio on Ruminal Fermentation and Microorganisms Using In vitro Gas Production Technique
}

\author{
S. Polyorach, M. Wanapat*, and A. Cherdthong \\ Tropical Feed Resources Research and Development Center (TROFREC), Department of Animal Science, \\ Faculty of Agriculture, Khon Kaen University, Khon Kaen 40002, Thailand
}

\begin{abstract}
The objective of this study was to determine the effects of protein sources and roughage (R) to concentrate (C) ratio on in vitro fermentation parameters using a gas production technique. The experimental design was a $2 \times 5$ factorial arrangement in a completely randomized design (CRD). Factor A was 2 levels of protein sources yeast fermented cassava chip protein (YEFECAP) and soybean meal $(\mathrm{SBM})$ and factor B was 5 levels of roughage to concentrate (R:C) ratio at 80:20, 60:40, 40:60, 20:80, and 0:100, respectively. Rice straw was used as a roughage source. It was found that gas production from the insoluble fraction (b) of YEFECAP supplemented group was significantly higher $(\mathrm{p}<0.05)$ than those in SBM supplemented group. Moreover, the intercept value $(a)$, gas production from the insoluble fraction (b), gas production rate constants for the insoluble fraction (c), potential extent of gas production $(\mathrm{a}+\mathrm{b})$ and cumulative gas production at $96 \mathrm{~h}$ were influenced $(\mathrm{p}<0.01)$ by $\mathrm{R}: \mathrm{C}$ ratio. In addition, protein source had no effect $(\mathrm{p}>0.05)$ on ether in vitro digestibility of dry matter (IVDMD) and organic (IVOMD) while R:C ratio affected the IVDMD and IVOMD ( $<<0.01$ ). Moreover, YEFECAP supplanted group showed a significantly increased ( $\mathrm{p}<0.05)$ total VFA and $\mathrm{C}_{3}$ while $\mathrm{C}_{2}, \mathrm{C}_{2}: \mathrm{C}_{3}$ and $\mathrm{CH}_{4}$ production were decreased when compared with SBM supplemented group. In addition, a decreasing $\mathrm{R}: \mathrm{C}$ ratio had a significant effect ( $\mathrm{p}<0.05)$ on increasing total VFA, $\mathrm{C}_{3}$ and $\mathrm{NH}_{3}-\mathrm{N}$, but decreasing the $\mathrm{C}_{2}, \mathrm{C}_{2}: \mathrm{C}_{3}$ and $\mathrm{CH}_{4}$ production ( $\mathrm{p}<0.01$ ). Furthermore, total bacteria, Fibrobacter succinogenes, Ruminococcus flavefaciens and Ruminococcus albus populations in YEFECAP supplemented group were significantly higher $(\mathrm{p}<0.05)$ than those in the SBM supplemented group while fungal zoospores, methanogens and protozoal population remained unchanged $(p>0.05)$ as compared between the two sources of protein. Moreover, fungal zoospores and total bacteria population were significantly increased $(\mathrm{p}<0.01)$ while, F. succinogenes, $R$. flavefaciens, $R$. albus, methanogens and protozoal population were decreased $(\mathrm{p}<0.01)$ with decreasing R:C ratio. In conclusion, YEFECAP has a potential for use as a protein source for improving rumen fermentation efficiency in ruminants. (Key Words: Yeast Fermented Cassava Chip Protein (YEFECAP), R:C Ratio, Protein Source, Rumen Fermentation, In vitro Gas Production, Rice Straw)
\end{abstract}

\section{INTRODUCTION}

The security of livestock feed is been becoming critical in terms of both quantity and quality, particularly the protein sources, lack of which results in low productivity. Researchers have been trying to find alternative protein sources which could help to increase livestock productivity and efficiency (Wanapat and Rowlinson, 2007; Guglielmelli

\begin{tabular}{l}
\hline * Corresponding Author: Metha Wanapat. Tel: +66-43-202368, \\
Fax: +66-43-202368, E-mail: metha@kku.ac.th \\
Submitted May 27 2013; Accepted Aug. 18, 2013; Revised Sept. 14, 2013
\end{tabular} et al., 2010). Cassava (Manihot esculenta, Crantz) is widely grown in the tropical region (Wanapat, 2003), its' tuber is a good source of carbohydrate and the leaf as a protein supplement in ruminants (Devendra, 1988; Wanapat, 2003). Cassava root is an excellent source of dietary energy in beef cattle diets (Wanapat and Khampa, 2007). However, it has a readily fermentable carbohydrate with a crude protein level that is low for ruminants (Wanapat, 2003). The process of protein enrichment of animal feed using microorganisms in a semi-solid culture to improve the nutritional value of ruminants feed has been evaluated (Oboh and Akindahinsi, 2003; Oboh, 2006; Aro, 2008). Incorporation of microbial

Copyright $@ 2014$ by Asian-Australasian Journal of Animal Sciences This is an open-access article distributed under the terms of the Creative Commons Attribution Non-Commercial License (http://creativecommons.org/licenses/by-nc/3.0/), which permits unrestricted non-commercial use, distribution, and reproduction in any medium, provided the original work is properly cited. 
additives such as a culture of Saccharomyces cerevisiae to the diet has become common practice in ruminant nutrition (Campanile et al., 2008). Boonnop et al. (2009) showed that using fermented cassava chip with yeast increased crude protein from 2 to $30.4 \% \mathrm{CP}$. Recently, Polyorach et al. (2012; 2013) reported that yeast fermented cassava chip protein (YEFECAP) could be prepared to increase crude protein level up to $47 \% \mathrm{CP}$. The beneficial use of YEFECAP has been investigated (Boonnop et al., 2010; Polyorach et al., 2010; Wanapat et al., 2011).

Roughage and concentrate type and ratio in complete feeds is of major importance for efficient utilization of dietary nutrients for production. Moderate amounts of concentrate in the diet can improve the utilization of fiber due to the better supply of fermentable organic matter, energy and nitrogen to rumen bacteria. Earlier work has shown that dietary protein degradation is increased by the inclusion of roughage in the diet (Schoeman et al., 1972), others have reported that protein degradation was either increased by cereal inclusion in the basal diet (Lindberg, 1981) or was unaffected by basal diet (Siddons and Paradine, 1981). Bach et al. (2005) indicated that the most important factors affecting utilization of dietary protein in the rumen included type of protein, carbohydrate and their interactions and the predominant microbial population in the rumen.

However, study of the use of YEFECAP as a protein source with various levels of roughage to concentrate (R:C) ratio have not yet been evaluated. Therefore, the objective of this study was to determine the affect of protein sources with various $\mathrm{R}: \mathrm{C}$ ratios on ruminal fermentation using an in vitro gas production technique.

\section{MATERIALS AND METHODS}

\section{Preparation of yeast-fermented cassava chip (YEFECAP)}

YEFECAP preparation was done according to the method of Polyorach et al. (2013) and some important details are as follows: activated yeast was prepared using 20 $\mathrm{g}$ of Bakers' yeast and $20 \mathrm{~g}$ cane sugar mixed with $100 \mathrm{~mL}$ distilled water, then mixed well and incubated at room temperature for $1 \mathrm{~h}(\mathrm{~A})$. Liquid media was prepared using 8 $\mathrm{g}$ molasses and $100 \mathrm{~mL}$ distilled water, followed by addition of $64 \mathrm{~g}$ urea, then adjusting the $\mathrm{pH}$ of the solution using $\mathrm{H}_{2} \mathrm{SO}_{4}$ to achieve a final $\mathrm{pH} 3.5$ to 5 (B). Mixed (A) and (B) at 1:1 ratio then flushed with air for $66 \mathrm{~h}$ at room temperature using an air pump $(600 \mathrm{~W})$. After $66 \mathrm{~h}$, the yeast medium solution twas mixed with cassava chips at a ratio of $1 \mathrm{~mL}: 1.3 \mathrm{~g}$, then fermented in solid state under shade for $72 \mathrm{~h}$, followed by sun-drying for $48 \mathrm{~h}$. The final product is stored in plastic bag for later use as an ingredient in the concentrate supplement.

\section{Experimental design and dietary treatments}

This study was conducted using an in vitro gas production technique at various incubation time intervals. The experimental design was a $2 \times 5$ factorial arrangement in a completely randomized design (CRD) with three replications per treatment. The treatments were two sources of protein in concentrate in which concentrate I used YEFECAP and concentrate II used soybean meal (SBM) as protein sources with five levels of R:C ratio at 80:20, 60:40, 40:60, 20:80, and 0:100, respectively. Rice straw was used as a roughage source. Samples of roughage and concentrates were dried at $60{ }^{\circ} \mathrm{C}$, then ground to pass a 1$\mathrm{mm}$ sieve (Cyclotech Mill, Tecator, Sweden) and used for chemical analysis and in the in vitro gas test. The samples were analyzed for dry matter (DM), ash and crude protein (CP) using the procedures of AOAC (1998), neutral detergent fiber (NDF) and acid detergent fiber (ADF) according to Van Soest et al. (1991).

\section{Animals and preparation of rumen inoculums}

Two, male 2-year-old, rumen fistulated dairy steers with an initial BW of $280 \pm 15.0 \mathrm{~kg}$ were used as rumen fluid donors. Dairy steer rumen fluid was collected from animals fed with concentrate $(18.0 \% \mathrm{CP}$ and $80.6 \% \mathrm{TDN})$ at $0.5 \%$ of $\mathrm{BW}$ in to equal portions, at $07.00 \mathrm{~h}$ and $16.00 \mathrm{~h}$ and rice straw was fed on ad libitum basis. The animals were kept in individual pens and clean fresh water and mineral blocks were offered as free choice. The animals received the diets for $20 \mathrm{~d}$ before the rumen fluid was collected. On d 20, $1,000 \mathrm{~mL}$ rumen liquor was obtained from each animal before the morning feeding. The rumen fluid was filtered through four layers of cheesecloth into pre-warmed thermo flasks and then transported to the laboratory.

\section{In vitro fermentation of substrates}

Samples of each total mixed substrate $(200 \mathrm{mg})$, following respective treatments were weighed into $50 \mathrm{~mL}$ serum bottles. For each treatment, three replications were prepared. Ruminal fluid from each animal was mixed with the artificial saliva solution of Menke and Steingass (1988) in a proportion $2: 1(\mathrm{~mL} / \mathrm{mL})$ at $39^{\circ} \mathrm{C}$ under continuous flushing with $\mathrm{CO}_{2}$. Thirty milliliters of rumen inoculum mixture were added into each bottle under $\mathrm{CO}_{2}$ flushing. Bottles were sealed with rubber stoppers and aluminium caps and incubated at $39^{\circ} \mathrm{C}(96 \mathrm{~h})$ for in vitro gas test. Thirty minutes after starting the incubation, the bottles were gently mixed and then mixed three times every $3 \mathrm{~h}$. For each sampling time, three bottles containing only the rumen inocula were included within each run and the mean gas production values of these bottles were used as blanks. The blank values were subtracted from each measured value to give the net gas production. 


\section{Sample and analysis}

During the incubation, data of gas production was measured immediately after incubation at $0,2,4,6,8,12$, $18,24,48,72$, and $96 \mathrm{~h}$ by using a pressure transducer and a calibrated syringe. Cumulative gas production data were fitted to the model of Orskov and McDonald (1979) as follows:

$$
y=a+b\left(1-e^{(-c t)}\right)
$$

Where $\mathrm{a}=$ the gas production from the immediately soluble fraction, $\mathrm{b}=$ the gas production from the insoluble fraction, $\mathrm{c}=$ the gas production rate constant for the insoluble fraction $(b), t=$ incubation time, $(a+b)=$ the potential extent of gas production. $y=$ gas produced at time " $t$ ".

Inoculum ruminal fluid was sampled at $0,4,6,12$, and $24 \mathrm{~h}$ post inoculations. Rumen fluid samples were then filtered through four layers of cheesecloth. Samples were divided into 3 portions; the first portion was centrifuged at $16,000 \times g$ for $15 \mathrm{~min}$, and the supernatant was stored at $-20^{\circ} \mathrm{C}$ before $\mathrm{NH}_{3}-\mathrm{N}$ analysis using the micro-Kjeldahl methods (AOAC, 1998) and VFA analysis using HPLC (Samuel et al., 1997). The second portion was fixed with $10 \%$ formalin solution in a sterilized $0.9 \%$ saline solution for a total direct count of bacteria, protozoa and fungi made by the methods of Galyean (1989) based on the use of a hemocytometer (Boeco, Hamburg, Germany). The final portion was stored at $-20^{\circ} \mathrm{C}$ for DNA extraction ( $\mathrm{Yu}$ and Morrison, 2004).

In vitro degradability was determined after termination of incubation, when the contents were filtered through preweighed Gooch crucibles and residual dry matter was estimated. The percent loss in weight was determined and presented as in vitro dry matter degradability (IVDMD). The dried feed sample and residue left from above was ashed at $550^{\circ} \mathrm{C}$ for determination of in vitro organic matter degradability (IVOMD) (Tilley and Terry, 1963). Calculation of ruminal methane $\left(\mathrm{CH}_{4}\right)$ production using VFA proportions was made according to Moss et al. (2000) and as follows:

$$
\begin{aligned}
& \mathrm{CH}_{4} \text { production } \\
& =0.45 \text { (acetate) }-0.275 \text { (propionate) }+0.4 \text { (butyrate) }
\end{aligned}
$$

\section{Rumen microbial population}

Community deoxyribonucleic acids (DNA) extraction: Community DNA was extracted from $0.5 \mathrm{~g}$ of rumen content (fluid and digesta) by the $\mathrm{RBB}+\mathrm{C}$ method ( $\mathrm{Yu}$ and Morrison, 2004). In brief, the $\mathrm{RBB}+\mathrm{C}$ method employs two rounds of bead beating in the presence of $\mathrm{NaCl}$ and SDS, followed by sequential ammonium acetate and isopropanal precipitations. The precipitated nucleic acids were then treated with RNase A and proteinase $\mathrm{K}$, and the DNA was purified using columns from QIAgen DNA Mini Stool Kit (QIAGEN, Valencia, CA).

Primers and polymerase chain reaction $(P C R)$ : The targeted bacteria were total bacteria, the three predominant cellulolytic bacteria (Fibrobactor succinogenes, Ruminococcus flavefaciens and Ruminococcus albus), methanogens and protozoa. Primers for F. succinogenes, Fs219f (5'GGT ATG GGA TGA GCT TGC-3') and Fs654r (5'-GCC TGC CCC TGA ACT ATC- 3'), were selected to allow amplification (446-bp product) of all $10 \mathrm{~F}$. succinogenes strains deposited in GenBank. For $R$. albus primers, Ra1281f (5'-CCC TAA AAG CAG TCT TAG TTC G-3') and Ra1439r (5' CCT CCT TGC GGT TAG AAC A3') (175-bp product). R. flavefaciens primers, Rf154f (5'TCT GGA AAC GGA TGG TA-3') and Rf425r (5'- CCT TTA AGA CAG GAG TTT ACA A-3'), were also selected to allow species-specific amplification (295 bp) of all seven $R$. flavefaciens strains deposited in GenBank. All these primer sets were previously published by Koike and Kobayashi (2001). For methanogens primers, forward primers (5'-TTCGGTGGATCDCARAGRGC-3') and reverse primers (5'-GBARGTCGW- AWCCGTAGAATCC $\left.-3^{\prime}\right)$ were employed as described by Denman et al. (2005). PCR conditions for $F$. succinogenes were as follows: $30 \mathrm{~s}$ at $94^{\circ} \mathrm{C}$ for denaturing, $30 \mathrm{~s}$ at $60^{\circ} \mathrm{C}$ for annealing and $30 \mathrm{~s}$ at $72^{\circ} \mathrm{C}$ for extension (48 cycles), except for $9 \mathrm{~min}$ denaturation in the first cycle and 10 min extension in the last cycle. Amplification of $16 \mathrm{~S}$ rRNA for the other two species was carried out similarly except an annealing temperature of $55^{\circ} \mathrm{C}$ was used. The PCR conditions for methanogens were as follows; $30 \mathrm{~s}$ at $94^{\circ} \mathrm{C}$ for denaturing, $30 \mathrm{~s}$ at $58^{\circ} \mathrm{C}$ for annealing and $90 \mathrm{~s}$ at $72^{\circ} \mathrm{C}$ for extension (35 cycles) (Wright et al., 2004) and ruminal protozoa was described by Sylvester et al. (2004).

Real-time PCR: The targeted bacteria were cellulolytic bacteria (F. succinogenes, $R$. albus, and $R$. flavefaciens) and methanogens. To establish a quantitative assay, we amplified target 16s rDNA of each species by using specific primers and PCR conditions as described previously, the purified DNA was quantified by spectrophotometry by comparing the products with serial 10-fold dilutions from 101 to 108 DNA copies of the previously quantified DNA standards. Real-time PCR amplification and detection were performed in a Chromo 4TM system (Bio-Rad, USA). In brief, Biostools QuantiMix Easy SYG Kit was used for PCR amplification. Samples were assayed in duplicate in a $20 \mu \mathrm{L}$ reaction mixture contained 4 to $6 \mathrm{mM} \mathrm{MgCl}_{2}, 10 \mu \mathrm{L}$ of Mastermix (including; Taq DNA polymerase, reaction buffer, dNTP mixture, $\mathrm{MgCl}_{2}$ and SybrGreen), $2 \mu \mathrm{L}$ of DNA template, and $0.8 \mu \mathrm{L}$ of each primer $(10 \mu \mathrm{M} / \mu \mathrm{L})$. 


\section{Statistical analysis}

All data were analyzed as a $2 \times 5$ factorial arrangement in a Completely randomized design (CRD) using the PROC GLM of SAS (1998). Data were analyzed using the model:

$$
\mathrm{Y}_{\mathrm{ij}}=\mu+\mathrm{A}_{\mathrm{i}}+\mathrm{B}_{\mathrm{j}}+\mathrm{AB}_{\mathrm{ij}}+\varepsilon_{\mathrm{ij}}
$$

Where: $Y=$ observations; $\mu=$ overall mean; $A_{i}=$ effect of factor $\mathrm{A}$ (protein sources, $\mathrm{i}=1$ to 2 ); $\mathrm{B}_{\mathrm{j}}=$ effect of factor $B$ (level of roughage to concentrate $(R: C)$ ratio, $j=1$ to 5 ), $\mathrm{AB}_{\mathrm{ij}}=$ interaction between factor $\mathrm{A}$ and $\mathrm{B}$, and $\varepsilon_{\mathrm{ij}}=$ the residual effect. Multiple comparisons among treatment means were performed by Duncan's New Multiple Range Test (DMRT) (Steel and Torrie, 1980). Differences among means with $\mathrm{p}<0.05$ were accepted as representing statistically significant differences. Trend of R:C ratio level was performed by orthogonal polynomials comparison.

\section{RESULTS}

Feed ingredients and chemical compositions of concentrate I, concentrate II, YEFECAP and rice straw are presented in Table 1. Concentrate I, using YEFECAP while concentrate II using SBM as main protein sources. Chemical composition of both concentrate I and concentrate II were similar between treatments. YEFECAP product contained DM, OM, CP, NDF, and ADF at 89.2, 95.6, 46.7, 6.7, and 4.2\% DM, respectively. Rice straw was used as a roughage source which contained DM, OM, CP, NDF, and $\mathrm{ADF}$ at 90.2, 81.3, 2.9, 82.7, and 57.6\% DM, respectively.
Cumulative gas production for each of the substrate treatments is presented as gas production and the values for estimated parameters obtained from the kinetics of gas production models for substrates studied are given in Table 2. Protein source had no effect $(p>0.05)$ on the kinetics of gas production except the gas production in the insoluble fraction (b) of YEFECAP group was significantly higher $(\mathrm{p}<0.05)$ than in the SBM group. Moreover, the intercept value (a), gas production from the insoluble fraction (b), gas production rate constants for the insoluble fraction (c), potential extent of gas production $(a+b)$ and cumulative gas production at $96 \mathrm{~h}$ were influenced $(\mathrm{p}<0.01)$ by the $\mathrm{R}: \mathrm{C}$ ratio.

In addition, the effect of protein source and $\mathrm{R}: \mathrm{C}$ ratio on in vitro degradability is presented in Table 2. Protein source had no effect $(p>0.05)$ on either IVDMD or IVOMD while $\mathrm{R}: \mathrm{C}$ ratio increased $(\mathrm{p}<0.01)$ IVDMD and IVOMD when the $\mathrm{R}: \mathrm{C}$ ratio level was decreasing and were highest in $\mathrm{R}: \mathrm{C}$ ratio at 40:60. However, there were interactions between protein sources and $\mathrm{R}: \mathrm{C}$ ratio on gas production kinetics and degradability.

The VFA, $\mathrm{NH}_{3}-\mathrm{N}$ and methane production $\left(\mathrm{CH}_{4}\right)$ are presented in Table 3. The results of the current study revealed that the YEFECAP fed group significantly increased $(p<0.01)$ total VFA and propionate $\left(C_{3}\right)$ while decreased $(\mathrm{p}<0.05)$ acetate to propionate ratio $\left(\mathrm{C}_{2}: \mathrm{C}_{3}\right)$ and $\mathrm{CH}_{4}$ production when compared with SBM fed group. However, with a decreasing $\mathrm{R}: \mathrm{C}$ ratio level, total VFA, $\mathrm{C}_{3}$ and $\mathrm{NH}_{3}-\mathrm{N}$ were increased $(\mathrm{p}<0.05)$ while $\mathrm{C}_{2}, \mathrm{C}_{2}: \mathrm{C}_{3}$ and $\mathrm{CH}_{4}$ production were decreased $(\mathrm{p}<0.05)$. Moreover, there

Table 1. Feed ingredients composition of dietary treatments used in the experiment

\begin{tabular}{|c|c|c|c|c|}
\hline Item & ${\text { Concentrate } \mathrm{I}^{1}}^{1}$ & Concentrate $\mathrm{II}^{2}$ & YEFECAP $^{3}$ & Rice straw \\
\hline \multicolumn{5}{|l|}{ Ingredient, $\mathrm{g} / \mathrm{kg}$ dry matter } \\
\hline Cassava chip & 62.2 & 62.2 & & \\
\hline Rice bran & 10.5 & 8.5 & & \\
\hline YEEFECAP $^{3}$ & 20.0 & 0.0 & & \\
\hline Soybean meal & 0.0 & 22.0 & & \\
\hline Fat & 8.0 & 0.8 & & \\
\hline Molasses & 2.0 & 2.0 & & \\
\hline Urea & 2.0 & 2.0 & & \\
\hline Mineral mixture & 1.0 & 1.0 & & \\
\hline Sulfur & 0.5 & 0.5 & & \\
\hline Salt & 1.0 & 1.0 & & \\
\hline \multicolumn{5}{|l|}{ Chemical composition } \\
\hline Dry matter $(\mathrm{g} / \mathrm{kg})$ & 90.0 & 90.8 & 892 & 902 \\
\hline & & -- Dry matte & & \\
\hline Organic matter & 93.3 & 94.1 & 956 & 813 \\
\hline Crude protein & 17.9 & 18.2 & 467 & 29 \\
\hline Neutral detergent fiber & 12.1 & 11.0 & 67 & 827 \\
\hline Acid detergent fiber & 8.3 & 7.5 & 42 & 576 \\
\hline
\end{tabular}

${ }^{1}$ Concentrate I = Using YEFECAP as protein sources. ${ }^{2}$ Concentrate II = Using soybean meal (SBM) as protein sources.

${ }^{3}$ YEFECAP $=$ Yeast fermented cassava chip protein. 
Table 2. Effect of protein source with roughage to concentrate ratio on gas production kinetics and degradability from in vitro incubation with rumen fluid

\begin{tabular}{|c|c|c|c|c|c|c|c|c|}
\hline \multirow{2}{*}{ Protein source ${ }^{1}$} & \multirow{2}{*}{$\mathrm{R}: \mathrm{C}^{2}$ ratio } & \multicolumn{4}{|c|}{ Gas kenetics $^{3}$} & \multirow{2}{*}{$\begin{array}{c}\text { Gas }(96 \mathrm{~h}) \mathrm{mL} \\
/ 0.2 \mathrm{~g} \mathrm{DM} \text { substrate }\end{array}$} & \multicolumn{2}{|c|}{ In vitro degradability (\%) } \\
\hline & & $\mathrm{a}$ & $\mathrm{b}$ & $\mathrm{c}$ & $a+b$ & & IVDMD & IVOMD \\
\hline \multirow[t]{5}{*}{ YEFECAP } & $80: 20$ & $-6.4^{\mathrm{d}}$ & $75.9^{\mathrm{a}}$ & $0.07^{\mathrm{a}}$ & $69.5^{\mathrm{a}}$ & $67.2^{\mathrm{ab}}$ & $62.1^{\mathrm{ab}}$ & $67.3^{\mathrm{cd}}$ \\
\hline & $60: 40$ & $-4.5^{\mathrm{cd}}$ & $72.9^{\mathrm{ab}}$ & $0.06^{\mathrm{abc}}$ & $68.4^{\mathrm{a}}$ & $67.0^{\mathrm{ab}}$ & $65.2^{\mathrm{ab}}$ & $77.8^{\mathrm{abc}}$ \\
\hline & 40:60 & $-3.8^{\mathrm{bcd}}$ & $75.4^{\mathrm{a}}$ & $0.06^{\mathrm{bc}}$ & $71.6^{\mathrm{a}}$ & $71.0^{\mathrm{a}}$ & $71.7^{\mathrm{a}}$ & $86.0^{\mathrm{a}}$ \\
\hline & $20: 80$ & $-2.3^{\mathrm{abc}}$ & $69.8^{\mathrm{ab}}$ & $0.06^{\mathrm{bc}}$ & $67.5^{\mathrm{a}}$ & $68.0^{\mathrm{ab}}$ & $70.2^{\mathrm{ab}}$ & $73.3^{\mathrm{bcd}}$ \\
\hline & $0: 100$ & $-0.7^{\mathrm{a}}$ & $59.7^{\mathrm{c}}$ & $0.06^{\mathrm{cd}}$ & $59.0^{\mathrm{bc}}$ & $59.8^{\mathrm{bc}}$ & $60.1^{\mathrm{ab}}$ & $63.9^{\mathrm{d}}$ \\
\hline \multirow[t]{5}{*}{ SBM } & $80: 20$ & $-5.3^{\mathrm{cd}}$ & $71.5^{\mathrm{ab}}$ & $0.07^{\mathrm{a}}$ & $66.3^{\mathrm{ab}}$ & $64.5^{\mathrm{abc}}$ & $60.3^{\mathrm{ab}}$ & $64.7^{\mathrm{d}}$ \\
\hline & $60: 40$ & $-4.5^{\mathrm{cd}}$ & $71.3^{\mathrm{ab}}$ & $0.07^{\mathrm{ab}}$ & $66.7^{\mathrm{ab}}$ & $66.0^{\mathrm{ab}}$ & $64.6^{\mathrm{ab}}$ & $76.8^{\mathrm{abc}}$ \\
\hline & $40: 60$ & $-2.5^{\mathrm{abc}}$ & $72.3^{\mathrm{ab}}$ & $0.06^{\mathrm{bc}}$ & $69.7^{\mathrm{a}}$ & $70.0^{\mathrm{a}}$ & $69.7^{\mathrm{ab}}$ & $81.8^{\mathrm{ab}}$ \\
\hline & $20: 80$ & $-1.0^{\mathrm{ab}}$ & $67.2^{\mathrm{b}}$ & $0.06^{\mathrm{bc}}$ & $66.2^{\mathrm{ab}}$ & $67.0^{\mathrm{ab}}$ & $64.7^{\mathrm{ab}}$ & $67.7^{\mathrm{cd}}$ \\
\hline & $0: 100$ & $-1.1^{\mathrm{ab}}$ & $56.0^{c}$ & $0.05^{\mathrm{d}}$ & $54.9^{c}$ & $56.2^{\mathrm{c}}$ & $59.7^{\mathrm{b}}$ & $65.2^{\mathrm{d}}$ \\
\hline SEM & & 0.90 & 2.27 & 0.003 & 2.59 & 2.69 & 3.50 & 3.39 \\
\hline \multicolumn{9}{|l|}{ Comparison } \\
\hline Protein source & & ns & $*$ & ns & ns & ns & ns & $\mathrm{ns}$ \\
\hline $\mathrm{R}: \mathrm{C}$ ratio & & $* *$ & $* *$ & $* *$ & $* *$ & $* *$ & $*$ & $* *$ \\
\hline Interaction & & ns & ns & ns & ns & ns & ns & ns \\
\hline \multicolumn{9}{|c|}{ Orthogonal polynomial } \\
\hline R:C (linear) & & $* *$ & $* *$ & $* *$ & $* *$ & $*$ & ns & ns \\
\hline $\mathrm{R}: \mathrm{C}$ (quadratic) & & ns & $* *$ & ns & $* *$ & $* *$ & $* *$ & $* *$ \\
\hline $\mathrm{R}: \mathrm{C}$ (cubic) & & ns & ns & ns & ns & ns & ns & ns \\
\hline
\end{tabular}

${ }^{\mathrm{a}-\mathrm{d}}$ Value on the same row with different superscripts differ $(\mathrm{p}<0.05), * \mathrm{p}<0.05, * * \mathrm{p}<0.01$.

$\mathrm{ns}=$ Non-significant, $\mathrm{SEM}=$ Standard error of the mean, YEFECAP = Yeast fermented cassava chip protein, SBM = Soybean meal .

${ }^{1} \mathrm{PS}=$ Protein source in concentrate. ${ }^{2} \mathrm{R}: \mathrm{C}=$ Roughage:concentrate ratio.

${ }^{3} \mathrm{a}=$ The gas production from the immediately soluble fraction, $\mathrm{b}=$ The gas production from the insoluble fraction, $\mathrm{c}=$ The gas production rate constant for the insoluble fraction (b), $a+b=$ The gas potential extent of gas production.

Table 3. Effect of protein source with roughage to concentrate ratio on in vitro volatile fatty acids (VFA), ammonia nitrogen $\left(\mathrm{NH}_{3}-\mathrm{N}\right)$ and methane production $\left(\mathrm{CH}_{4}\right)$

\begin{tabular}{|c|c|c|c|c|c|c|c|c|}
\hline Protein source ${ }^{1}$ & $\begin{array}{l}\mathrm{R}: \mathrm{C}^{2} \\
\text { ratio }\end{array}$ & $\begin{array}{c}\text { Total VFA } \\
(\mathrm{mM} / \mathrm{L})\end{array}$ & $\mathrm{C}_{2}(\%)$ & $\mathrm{C}_{3}(\%)$ & $\mathrm{C}_{4}(\%)$ & $\mathrm{C}_{2}: \mathrm{C}_{3}$ ratio & $\begin{array}{c}\mathrm{NH}_{3}-\mathrm{N} \\
(\mathrm{mg} / \mathrm{dL})\end{array}$ & $\begin{array}{c}\mathrm{CH}_{4} \\
\text { production } \\
(\mathrm{mM} / \mathrm{L})\end{array}$ \\
\hline \multirow[t]{5}{*}{ YEFECAP } & $80: 20$ & $65.0^{\mathrm{ab}}$ & $60.5^{\text {bcd }}$ & $28.4^{\mathrm{abc}}$ & $10.8^{\mathrm{ab}}$ & $2.2^{\text {cde }}$ & $26.6^{\mathrm{a}}$ & $21.0^{\mathrm{cd}}$ \\
\hline & $60: 40$ & $64.1^{\mathrm{ab}}$ & $58.2^{\mathrm{cd}}$ & $30.2^{\mathrm{ab}}$ & $11.6^{\mathrm{ab}}$ & $2.0^{\mathrm{de}}$ & $24.5^{\mathrm{abc}}$ & $21.0^{\mathrm{cd}}$ \\
\hline & $40: 60$ & $68.5^{\mathrm{a}}$ & $57.2^{\mathrm{cd}}$ & $30.4^{\mathrm{ab}}$ & $12.4^{\mathrm{ab}}$ & $1.9^{\mathrm{de}}$ & $21.7^{\mathrm{bcd}}$ & $22.3^{c}$ \\
\hline & $20: 80$ & $65.0^{\mathrm{ab}}$ & $61.6^{\mathrm{bc}}$ & $28.4^{\mathrm{abc}}$ & $10.1^{\mathrm{ab}}$ & $2.2^{\mathrm{cd}}$ & $20.0^{\mathrm{cd}}$ & $23.9^{\mathrm{bc}}$ \\
\hline & $0: 100$ & $56.1^{\mathrm{bcd}}$ & $67.0^{\mathrm{a}}$ & $23.4^{\mathrm{de}}$ & $9.7^{\mathrm{b}}$ & $2.9^{\mathrm{a}}$ & $18.9^{\mathrm{d}}$ & $24.3^{\mathrm{bc}}$ \\
\hline \multirow[t]{5}{*}{ SBM } & $80: 20$ & $52.1^{\mathrm{cd}}$ & $56.1^{\mathrm{d}}$ & $32.4^{\mathrm{a}}$ & $11.5^{\mathrm{ab}}$ & $1.7^{\mathrm{e}}$ & $24.9^{\mathrm{ab}}$ & $18.8^{\mathrm{d}}$ \\
\hline & $60: 40$ & $57.1^{\text {bcd }}$ & $60.7^{\mathrm{cd}}$ & $25.3^{\text {cde }}$ & $14.1^{\mathrm{a}}$ & $2.4^{\mathrm{bc}}$ & $24.1^{\mathrm{abc}}$ & $22.0^{\mathrm{cd}}$ \\
\hline & $40: 60$ & $61.4^{\mathrm{abc}}$ & $61.5^{\mathrm{bc}}$ & $27.5^{\mathrm{bcd}}$ & $11.0^{\mathrm{ab}}$ & $2.2^{\mathrm{cd}}$ & $24.0^{\mathrm{abc}}$ & $24.5^{\mathrm{bc}}$ \\
\hline & $20: 80$ & $56.1^{\text {bcd }}$ & $65.6^{\mathrm{ab}}$ & $23.9^{\text {cde }}$ & $10.6^{\mathrm{ab}}$ & $2.8^{\mathrm{ab}}$ & $21.8^{\mathrm{bcd}}$ & $26.2^{\mathrm{ab}}$ \\
\hline & $0: 100$ & $50.1^{\mathrm{d}}$ & $66.4^{\mathrm{a}}$ & $21.6^{\mathrm{e}}$ & $12.0^{\mathrm{ab}}$ & $3.1^{\mathrm{a}}$ & $19.8^{\mathrm{dc}}$ & $28.8^{\mathrm{a}}$ \\
\hline SEM & & 2.89 & 1.42 & 1.33 & 1.17 & 0.12 & 1.39 & 1.01 \\
\hline \multicolumn{9}{|l|}{ Comparison } \\
\hline Protein source ${ }^{1}$ & & $* *$ & ns & $*$ & $\mathrm{~ns}$ & $*$ & $\mathrm{~ns}$ & $*$ \\
\hline $\mathrm{R}: \mathrm{C}$ ratio $^{2}$ & & $*$ & $* *$ & $* *$ & ns & $* *$ & $* *$ & $* *$ \\
\hline Interaction & & $\mathrm{ns}$ & $*$ & $*$ & ns & ns & ns & $\mathrm{ns}$ \\
\hline \multicolumn{9}{|c|}{ Orthogonal polynomial } \\
\hline $\mathrm{R}: \mathrm{C}$ (linear) & & $\mathrm{ns}$ & $* *$ & $* *$ & ns & $* *$ & $* *$ & $* *$ \\
\hline $\mathrm{R}: \mathrm{C}$ (quadratic) & & $* *$ & $*$ & ns & ns & $* *$ & $\mathrm{~ns}$ & $\mathrm{~ns}$ \\
\hline $\mathrm{R}: \mathrm{C}$ (cubic) & & ns & ns & ns & ns & ns & $\mathrm{ns}$ & $\mathrm{ns}$ \\
\hline
\end{tabular}

${ }^{\mathrm{a}-\mathrm{e}}$ Value on the same row with different superscripts $\operatorname{differ}(\mathrm{p}<0.05),{ }^{*} \mathrm{p}<0.05, * * \mathrm{p}<0.01$.

$\mathrm{ns}=$ Non-significant, $\mathrm{SEM}=$ Standard error of the mean, YEFECAP = Yeast fermented cassava chip protein, $\mathrm{SBM}=$ Soybean meal.

${ }^{1} \mathrm{PS}=$ Protein source in concentrate. ${ }^{2} \mathrm{R}: \mathrm{C}=$ Roughage:concentrate ratio.

${ }^{3}$ Calculated according to Moss et al. (2000) $\mathrm{CH}_{4}$ production $=0.45\left(\mathrm{C}_{2}\right)-0.275\left(\mathrm{C}_{3}\right)+0.4\left(\mathrm{C}_{4}\right)$. 
were interactions between protein sources and $\mathrm{R}: \mathrm{C}$ ratio which affected $(\mathrm{p}<0.05)$ on $\mathrm{C} 2, \mathrm{C} 3$ and $\mathrm{C}_{2}$ : $\mathrm{C}_{3}$.

Effect of protein source and $\mathrm{R}: \mathrm{C}$ ratio on ruminal microorganisms is presented in Table 4. The results revealed that the bacterial population in YEFECAP fed group were significantly higher $(p<0.01)$ than in the SBM group while fungal zoospores and protozoal population were not significantly different $(p>0.05)$ between the two sources of protein. However, with a decreasing R:C ratio level, bacterial and fungal zoospores populations were increased $(\mathrm{p}<0.01)$ while the protozoal population was decreased $(p<0.01)$. External standards for real-time PCR were prepared from a simulated rumen matrix. For each standard, linear regressions derived from the threshold cycle $(\mathrm{C}[\mathrm{T}])$ of each DNA dilution versus the $\log$ quality were calculated. Logarithms of the DNA concentration (copies $/ \mathrm{mL}$ ) were plotted against the calculated means, obtaining straight line equations $\mathrm{y}=-0.3541 \mathrm{x}+11.29, \mathrm{y}=$ $-0.3094 x+12.48, y=-0.3766 x+10.38, y=-0.3293 x+11.03$, $y=-0.3279 x+11.23$ and $y=-0.2176 x+10.66$ (where $y$ is the $\log$ of DNA concentration and $\mathrm{x}$ is the $\mathrm{Ct}$ ), with a linear correlation coefficient $\left(\mathrm{r}^{2}\right)$ of $0.986,0.985,0.996,0.993$, 0.998 , and 0.992 for total bacteria, $F$. succinogenes, $R$. flavefaciens, $R$. albus, methanogens and protozoa, respectively. The accuracy of each real-time PCR was validated by quantifying known numbers of target species templates (total bacteria, $F$. succinogenes, $R$. flavefaciens, $R$. albus, methanogens and protozoa) and is shown in Table 4. It was shown that the interaction between protein source and $\mathrm{R}: \mathrm{C}$ ratio did not affect populations of protozoa, total bacteria, methanogens and predominant cellulolytic bacteria except $R$. flavefacieus. Whereas, total bacteria, $F$. succinogenes, $R$. flavefacieus, and $R$. albus in the YEFECAP supplemented group were significantly higher $(p<0.05)$ than in the SBM supplemented group. Moreover, when increasing concentrate level, total bacterial and fungal zoospores population were increased $(\mathrm{p}<0.01)$ and the highest in R:C ratio was found at 40:60, while, $F$. succinogenes, $R$. flavefaciens, $R$. albus, protozoal population and methanogens were significantly decreased $(\mathrm{p}<0.01)$ with increasing concentrate level.

\section{DISCUSSION}

Under this experiment, the rice straw that was used as a main roughage source contained $2.9 \% \mathrm{CP}$ (Table 1). This value was similar to those values reported by Wanapat et al. (2009). Moreover, crude protein of YEFECAP was similar to the value reported earlier by Polyorach et al. (2012; 2013) while this value was relatively higher than those reported by Boonnop et al. (2009) and Wanapat et al. (2011) which could be due to the differences of proportion of urea

Table 4. Effect of protein source with roughage to concentrate ratio on ruminal microorganism

\begin{tabular}{|c|c|c|c|c|c|c|c|c|c|c|}
\hline \multirow{2}{*}{$\begin{array}{l}\text { Protein } \\
\text { source }^{1}\end{array}$} & \multirow[b]{2}{*}{$\begin{array}{l}\mathrm{R}: \mathrm{C}^{2} \\
\text { ratio }\end{array}$} & \multicolumn{3}{|c|}{ Total direct count (cell/mL) } & \multicolumn{6}{|c|}{ Real-time PCR technique (copies/mL of incubation) } \\
\hline & & $\begin{array}{c}\text { Bacteria } \\
\left(\times 10^{7}\right)\end{array}$ & $\begin{array}{l}\text { Protozoa } \\
\left(\times 10^{5}\right)\end{array}$ & $\begin{array}{c}\text { Fungal } \\
\text { zoospores } \\
\left(\times 10^{5}\right)\end{array}$ & $\begin{array}{l}\text { Protozoa } \\
\left(\times 10^{5}\right)\end{array}$ & $\begin{array}{c}\text { Total } \\
\text { bacteria } \\
\left(\times 10^{9}\right)\end{array}$ & $\begin{array}{l}\text { Methanogens } \\
\qquad\left(\times 10^{6}\right)\end{array}$ & $\begin{array}{c}F . \\
\text { succinogenes } \\
\left(\times 10^{7}\right)\end{array}$ & $\begin{array}{c}R . \\
\text { flavefaciens } \\
\left(\times 10^{6}\right)\end{array}$ & $\begin{array}{c}\text { R. albus } \\
\left(\times 10^{6}\right)\end{array}$ \\
\hline \multirow[t]{5}{*}{ YEFECAP } & $80: 20$ & $5.5^{\mathrm{ef}}$ & $1.8^{\mathrm{b}}$ & $2.8^{\mathrm{ef}}$ & $2.8^{\mathrm{ab}}$ & $3.8^{\mathrm{bcd}}$ & $2.6^{\mathrm{ab}}$ & $5.1^{\mathrm{a}}$ & $5.9^{\mathrm{a}}$ & $5.1^{\mathrm{a}}$ \\
\hline & $60: 40$ & $7.6^{\mathrm{bcd}}$ & $1.7^{\mathrm{b}}$ & $4.0^{\text {cde }}$ & $2.5^{\mathrm{ab}}$ & $4.4^{\mathrm{ab}}$ & $2.4^{\mathrm{abc}}$ & $4.3^{\mathrm{ab}}$ & $4.6^{\mathrm{b}}$ & $4.0^{\mathrm{ab}}$ \\
\hline & 40:60 & $10.8^{\mathrm{a}}$ & $1.7^{\mathrm{b}}$ & $6.2^{\mathrm{a}}$ & $1.8^{\mathrm{b}}$ & $4.9^{\mathrm{a}}$ & $1.6^{\mathrm{cd}}$ & $3.1^{\mathrm{cd}}$ & $3.1^{\mathrm{cd}}$ & $3.1^{\mathrm{bcd}}$ \\
\hline & 20:80 & $9.3^{\mathrm{ab}}$ & $2.1^{\mathrm{b}}$ & $5.8^{\mathrm{a}}$ & $1.9^{\mathrm{ab}}$ & $3.5^{\text {cde }}$ & $1.8^{\mathrm{cd}}$ & $2.2^{\mathrm{de}}$ & $2.3^{\mathrm{e}}$ & $1.9^{\mathrm{de}}$ \\
\hline & $0: 100$ & $7.9^{\mathrm{bc}}$ & $3.0^{\mathrm{a}}$ & $5.0^{\mathrm{abcd}}$ & $2.1^{\mathrm{ab}}$ & $1.5^{\mathrm{f}}$ & $1.1^{\mathrm{d}}$ & $0.8^{\mathrm{f}}$ & $0.8^{\mathrm{f}}$ & $0.5^{\mathrm{f}}$ \\
\hline \multirow[t]{5}{*}{ SBM } & $80: 20$ & $4.3^{\mathrm{f}}$ & $1.8^{\mathrm{b}}$ & $2.6^{\mathrm{f}}$ & $3.2^{\mathrm{a}}$ & $3.3^{\mathrm{de}}$ & $2.9^{\mathrm{a}}$ & $4.3^{\mathrm{ab}}$ & $3.8^{\mathrm{bc}}$ & $3.6^{\mathrm{bc}}$ \\
\hline & $60: 40$ & $5.8^{\mathrm{def}}$ & $1.9^{\mathrm{b}}$ & $3.9^{\mathrm{de}}$ & $2.8^{\mathrm{ab}}$ & $3.8^{\mathrm{cd}}$ & $2.6^{\mathrm{ab}}$ & $3.8^{\mathrm{bc}}$ & $2.8^{\mathrm{de}}$ & $2.6^{\text {cde }}$ \\
\hline & $40: 60$ & $7.9^{\mathrm{bc}}$ & $2.0^{\mathrm{b}}$ & $5.3^{\mathrm{ab}}$ & $2.0^{\mathrm{ab}}$ & $4.2^{\mathrm{bc}}$ & $1.7^{\mathrm{cd}}$ & $2.9^{\mathrm{cd}}$ & $2.0^{\mathrm{e}}$ & $2.1^{\mathrm{de}}$ \\
\hline & $20: 80$ & $6.9^{\text {cde }}$ & $2.2^{\mathrm{b}}$ & $5.2^{\mathrm{abc}}$ & $2.2^{\mathrm{ab}}$ & $3.0^{\mathrm{e}}$ & $1.9^{\mathrm{bcd}}$ & $1.8^{\mathrm{e}}$ & $1.1^{\mathrm{f}}$ & $1.5^{\mathrm{ef}}$ \\
\hline & $0: 100$ & $5.0^{\mathrm{ef}}$ & $3.3^{\mathrm{b}}$ & $4.2^{\mathrm{bcd}}$ & $2.4^{\mathrm{ab}}$ & $1.2^{\mathrm{f}}$ & $1.2^{\mathrm{d}}$ & $0.5^{\mathrm{f}}$ & $0.5^{\mathrm{f}}$ & $0.6^{\mathrm{f}}$ \\
\hline SEM & & 0.59 & 0.26 & 0.37 & 0.38 & 0.19 & 0.24 & 0.31 & 0.25 & 0.35 \\
\hline \multicolumn{11}{|l|}{ Comparison } \\
\hline \multicolumn{2}{|c|}{ Protein source ${ }^{1}$} & $* *$ & ns & ns & ns & $* *$ & $\mathrm{~ns}$ & $*$ & $* *$ & $* *$ \\
\hline \multicolumn{2}{|l|}{$\mathrm{R}: \mathrm{C}$ ratio $^{2}$} & $* *$ & $* *$ & $* *$ & $* *$ & $* *$ & $* *$ & $* *$ & $* *$ & $* *$ \\
\hline \multicolumn{2}{|l|}{ Interaction } & $\mathrm{ns}$ & $\mathrm{ns}$ & ns & $\mathrm{ns}$ & ns & $\mathrm{ns}$ & ns & $*$ & ns \\
\hline \multicolumn{11}{|c|}{ Orthogonal polynomial } \\
\hline \multicolumn{2}{|c|}{$\mathrm{R}: \mathrm{C}$ (linear) } & $* *$ & $* *$ & $* *$ & $*$ & $* *$ & $* *$ & $* *$ & $* *$ & $* *$ \\
\hline \multicolumn{2}{|c|}{$\mathrm{R}: \mathrm{C}$ (quadratic) } & $* *$ & $* *$ & $* *$ & $\mathrm{~ns}$ & $* *$ & ns & ns & $\mathrm{ns}$ & ns \\
\hline \multicolumn{2}{|c|}{$\mathrm{R}: \mathrm{C}$ (cubic) } & $\mathrm{ns}$ & ns & ns & $\mathrm{ns}$ & ns & ns & ns & $\mathrm{ns}$ & ns \\
\hline
\end{tabular}

${ }^{\mathrm{a}-\mathrm{f}}$ Value on the same row with different superscripts differ $(\mathrm{p}<0.05), * \mathrm{p}<0.05, * * \mathrm{p}<0.01$

$\mathrm{ns}=$ Non-significant, SEM $=$ Standard error of the mean.

${ }^{1} \mathrm{PS}=$ Protein source in concentrate. ${ }^{2} \mathrm{R}: \mathrm{C}=$ Roughage: concentrate ratio 
and molasses used in the liquid medium, as well as the proportions of yeast fermented liquid and cassava chips used.

The present result revealed that gas production in the insoluble fraction (b) in YEFECAP supplemented group was significantly higher $(\mathrm{p}<0.05)$ than in SBM supplemented group. This was probably due to YEFECAP promoting growth of rumen cellulolytic bacteria and lactate-utilizing bacteria. Yeast may also have stimulated bacterial growth through removal of oxygen that occurs in ruminal fluid and in that way can prevent toxicity to the ruminal anaerobes (Chaucheyras-Durand et al., 2008; Doto and Liu, 2011). This will in turn increase polysaccharidase and glycoside-hydrolase activities towards lignified plant tissues, "unlocking" the fiber's digestible fraction (hemicellulose and cellulose), which will be digested by the cellulolytic microorganisms. These results were similar to the finding of Wanapat et al. (2011) that YEFECAP can fully replace SBM in concentrate for dairy cows and improved rumen fermentation, dry matter intake, nutrient digestibility, milk production and composition, as well as the improving the economic return in early lactating dairy crossbreds.

Gas kinetics and cumulative gas production were influenced by different $\mathrm{R}: \mathrm{C}$ ratios, especially the constant rate (c) and in vitro degradability of both $\mathrm{DM}$ and $\mathrm{OM}$ which were increased $(p<0.01)$ with an increasing level of concentrate and the highest was in $\mathrm{R}: \mathrm{C}$ at 40:60. These could be due to increased concentrate level that would provide more readily available energy, enhancing corresponding of microbes, consequently, increased degradability. The stimulatory effect of concentrate feeds on the ruminal microflora is well documented and, according to Hungate (1966) who reported that a pronounced effect in the rumen wamore readily achieved from carbohydrate than from forages. These findings were in agreement with Lunsin and Wanapat (2010) and Anantasook and Wanapat (2012). Moreover, Cherdthong et al. (2010) reported that the value for NDF and ADF were decreased particularly with the highest level of concentrate. However, a high level of concentrate may lower the $\mathrm{pH}$ and impact on the microbial population (Slyter, 1976).

The level of $\mathrm{NH}_{3}-\mathrm{N}$ concentration increased with an increasing concentrate level and the values ranged from 19.8 to $26.6 \mathrm{mg} / \mathrm{dL}$ which were similar to the values reported by Wanapat and Pimpa (1999) (15 to $30 \mathrm{mg} / \mathrm{dL}$ in the rumen).

In addition, YEFECAP as a protein source group significantly increased $(\mathrm{p}<0.05)$ total VFA and $\mathrm{C}_{3}$ while decreased $(p<0.05) \quad C_{2}: C_{3}$. These results suggested that YEFECAP is a good source of protein as Wanapat et al. (2011) also reported that total VFA and $C_{3}$ production were significantly increased, consequently, $\mathrm{C}_{2}: \mathrm{C}_{3}$ ratio was reduced in the $100 \%$ YEFECAP supplemented group. Moreover, this was probably due to some effects of the yeast contained in YEFECAP that stimulated the growth and metabolism of rumen microorganisms especially lactate-utilizing bacteria, such as Megasphaera elsdenii or Selenomonas ruminantium (Lynch and Martin, 2002). In addition, yeast could supply different growth factors, such as amino acids, peptides, vitamins, and organic acids, essential for the ruminal bacterial growth (ChaucheyrasDurand et al., 2008), hence enhancing VFA concentration and reducing $\mathrm{C}_{2}: \mathrm{C}_{3}$ proportion (Oeztuerk, 2009).

Furthermore, total VFA and $\mathrm{C}_{3}$ were increased while $\mathrm{C}_{2}$, and $\mathrm{C}_{2}: \mathrm{C}_{3}$ ratio were decreased with a increasing concentrate level. Similar to the current experiment, an enhanced propionate concentration was found in the rumen of cows fed high-grain as compared with high-forage diets (Sutton et al., 2003; Calabrò et al., 2008; Zicarelli et al., 2011). Surprisingly, Moorby et al. (2006) reported linear increases in total VFA and butyrate concentrations and a decrease in acetate with increasing proportion of concentrate in dietary DM, but the concentration of propionate was not affected. Calsamiglia et al. (2008) and Cherdthong et al. (2010) also reported that high concentrate diet fermentation resulted in a greater molar proportion of ruminal propionate. The inverse of relationship between $\mathrm{C}_{2}: \mathrm{C}_{3}$ ratio and amount of concentrate in the diet has often been explained by the tendency of fiber fermenting bacteria to produce $\mathrm{C}_{2}$ and starch fermenting bacteria to produce $\mathrm{C}_{3}$ (Slyter, 1976).

$\mathrm{CH}_{4}$ production in the rumen of YEFECAP supplemented group was lower $(p<0.05)$ when compared with SBM group. This could be due to the yeast in YEFECAP, probably affecting hydrogen metabolism in the rumen. Yeast also has the potential to alter the fermentation process in the rumen in a manner that reduces the formation of methane $\left(\mathrm{CH}_{4}\right)$ gas. Previously, Lynch and Martin (2002) reported a $20 \mathrm{mM} / \mathrm{L}$ reduction of methane production after $48 \mathrm{~h}$ of incubation of mixed rumen microorganisms in the presence of alfalfa and a live yeast product. McGinn et al. (2004) showed a $3 \%$ (g/ kg of DMI) decrease in in vivo $\mathrm{CH}_{4}$ production for one commercial yeast product. Moreover, $\mathrm{CH}_{4}$ production was also influenced by $\mathrm{R}: \mathrm{C}$ ratio, when an increasing concentrate level decreased $(p<0.01)$ methane production and the lowest value was at $0: 100$. This result might be due to an increased proportion of concentrate in the diet which changes ruminal VFAs concentrations in such a way that less $\mathrm{C}_{2}$ and more $\mathrm{C}_{3}$ is formed, and hence, the supply of hydrogen for methanogenesis is limited. In addition, concentrate feeding has been shown to reduce methane output by reducing the protozoal population (Van Soest, 1982; Iqbal et al., 2008). The amount of $\mathrm{CH}_{4}$ produced varies according with the type of diet (forage/concentrate) and the type of production system 
(intensive/extensive) (Moss et al., 2000; Aluwong et al., 2011). A positive response to high levels of starch-based concentrate (grains) on methane reduction has also been reported by others (Yan et al., 2000; Beauchemin and McGinn, 2005; Lovett et al., 2005; Poungchompu et al., 2009).

The results showed that the bacterial population in YEFECAP supplemented group was significantly higher $(\mathrm{p}<0.01)$ than in the SBM supplented group while fungal zoospores and protozoal population were not changed ( $>>0.05)$. These results related with the data from real-time PCR technique, regarding total bacteria, $F$. succinogenes, $R$. flavefacieus, and $R$. albus in the YEFECAP fed group which were significantly higher $(p<0.05)$ than in the SBM fed group which received a good protein source and the presence of yeast. Jouany (2006) reported that there were three main roles of yeast in the rumen that have been identified: i) Yeasts are source of nutrients for rumen microbes, ii) Yeasts could consume oxygen which is present in the microenvironment surrounding solid particles which have just been ingested into the rumen, iii) $S$. cerevisiae was able to out compete $S$. bovis for the utilization of sugars. These attributes create an optimal environment for rumen microbe activity especially cellulolytic bacteria. Additionally, S. cerevisiae leads to increased germination of zoospores from a rumen fungal strain of Neocallimastix frontalis as shown in an in vitro study (Chaucheyras et al., 1995). Callaway and Martin (1997) also showed that cellulolytic bacteria became established earlier, and remained at a high and stable level, even after a particularly stressful period when lambs, fitted with a rumen cannula, were fed an active dry yeast (ADY) daily. The increasing of cellulolytic bacteria was also mesured with real-time PCR as reported by Mosoni et al. (2007) who showed that proportions of $16 \mathrm{~S}$ ribosomal RNA of the three main cellulolytic bacterial species ( $F$. succinogenes, $R$. albus, and $R$. flavefaciens) increased in the rumen of sheep fed with yeast.

These results agreed with Boonnop et al. (2010) who found that YEFECAP could completely replace soybean meal and was beneficial to cattle in terms of efficiency of rumen fermentation, microbial protein synthesis, nitrogen retention and nutrient digestibilities. Polyorach et al. (2010) and Wanapat et al. (2011) later reported that increasing the level of YEFECAP in concentrate mixtures linearly increased the population of bacterial and fungal zoospores in the rumen while protozoa were not changed.

In addition, populations of fungal zoospores and total bacteria were significantly increased $(\mathrm{p}<0.01)$ with the highest levels found when the R:C ratio was at 40:60, while predominant cellulolytic bacteria ( $F$. succinogenes, $R$. flavefaciens, and $R$. albus), methanogens and protozoal population were decreased with a decreasing level in the
$\mathrm{R}: \mathrm{C}$ ratio. Decreasing of the three main cellulolytic bacterial populations in this study might be due to concentrate-rich diets. The influence of concentrate on fiber digestion and roughage utilization depends also on composition and ruminal fermentability of concentrate feed in the diet (Choi et al., 2003; Tafaj et al., 2005). Similarly to Cherdthong et al. (2010) who reported that R:C ratio of 100:0, 75:25, 50:50, and 25:75 could increase bacterial and fungal population while decreased numbers of the three main cellulolytic bacteria ( $F$. succinogenes, $R$. flavefaciens and $R$. albus) occurred with increasing concentrate levels. Wara-anu et al. (2000) reported that roughage- toconcentrate ratios of 100:0, 60:40, and 40:60 could decrease the cellulolytic bacterial population in swamp buffalo $\left(5.62 \times 10^{10}, \quad 4.06 \times 10^{10}\right.$, and $\left.4.57 \times 10^{10} \quad \mathrm{CFU} / \mathrm{mL}\right)$, respectively. Moreover, Anantasook and Wanapat (2012), using the in vitro gas production technique, also confirmed that the bacterial population was increased while cellulolytic bacterial and protozoal populations were decreased with increasing concentrate level.

\section{CONCLUSIONS}

Based on this study, it could be concluded that using YEFECAP as a protein source with R:C ratio of 40:60 could improve in vitro nutrient degradability and rumen fermentation by increasing total VFA, $\mathrm{C}_{3}$, fungal zoospores and total bacteria while $\mathrm{C}_{2}, \mathrm{C}_{2}: \mathrm{C}_{3}$ ratio, $\mathrm{CH}_{4}$ production, methanogens and protozoal population were decreased. These results reveal a potential use of YEFECAP as a protein source, leading to improving rumen fermentation efficiency and a possible productivity in ruminants.

\section{ACKNOWLEDGEMENTS}

The authors would like to express their most sincere thanks to the Tropical Feed Resources Research and Development Center (TROFREC), Department of Animal Science, Faculty of Agriculture, Khon Kaen University, Khon Kaen, Thailand, The Thailand Research Fund (TRF) through the Royal Golden Jubilee Ph. D. Scholarship Program for providing financial support of research and the use of research facilities.

\section{REFERENCES}

Aluwong, T., P. A. Wuyep, and L. Allam. 2011. Livestockenvironment interactions: Methane emissions from ruminants. Afr. J. Biotechnol. 10:1265-1269.

Anantasook, N. and M. Wanapat. 2012. Influence of rain tree pod meal supplementation on rice straw based diets using in vitro gas fermentation technique. Asian-Aust. J. Anim. Sci. 25:325334

Aro, S. O. 2008. Improve in the nutritive quality of cassava and its 
by-products through microbial fermentation. Afr. J. Biotechnol. 7:4789-4797.

Association of Official Analytical Chemists (AOAC). 1998. Official methods of analysis, vol. $2,16^{+}$edition. AOAC, Arlington, VA, USA

Bach, A., S. Calsamiglia, and M. D. Stern. 2005. Nitrogen metabolism in the rumen. J. Dairy Sci. 88 (E. Suppl.): E9-E21.

Beauchemin, K. A. and S. M. McGinn. 2005. Methane emissions from feedlot cattle fed barley or corn diets. J. Anim. Sci. 83:653-661.

Boonnop, K., M. Wanapat, N. Nontaso, and S. Wanapat. 2009. Enriching nutritive value of cassava root by yeast fermentation. Sci. Agric. 66:629-633.

Boonnop, K., M. Wanapat, and C. Navanukraw. 2010. Replacement of soybean meal by yeast fermented-cassava chip protein (YEFECAP) in concentrate diets fed on rumen fermentation, microbial population and nutrient digestibilities in ruminants. J. Anim. Vet. Adv. 9:1727-1734.

Calabrò, S., G. Moniello, V. Piccolo, F. Bovera, F. Infascelli, R. Tudisco, and M. I. Cutrignelli. 2008. Rumen fermentation and degradability in buffalo and cattle using the in vitro gas production technique. J. Anim. Physiol. Anim. Nutr. 92:356362.

Callaway, E. S. and S. A. Martin. 1997. Effects of a Saccharomyces cerevisiae culture on ruminal bacteria that utilize lactate and digest cellulose. J. Dairy Sci. 80:2035-2044.

Calsamiglia, S., P. W. Cardozo, A. Ferret, and A. Bach. 2008. Changes in rumen microbial fermentation are due to a combined effect of type of diet and pH. J. Anim. Sci. 86:702711.

Campanile, G., F. Zicarelli, D. Vecchio, C. Pacelli, G. Neglia, A. Balestrieri, R. Di Palo, and F. Infascelli. 2008. Effects of Saccharomyces cerevisiae on in vivo organic matter digestibility and milk yield in buffalo cows. Livest. Sci. 114:358-361.

Chaucheyras, F., G. Fonty, G. Bertin, J. M. Salmon, and P. Gouet. 1996. Effects of a strain of Saccharomyces cerevisiae (Levucell SC), a microbial additive for ruminants, on lactate metabolism in vitro. Can. J. Microbiol. 42:927-933.

Chaucheyras-Durand, F., N. D. Walker, and A. Bach. 2008. Effects of active dry yeasts on the rumen microbial ecosystem: Past, present and future. Anim. Feed Sci. Technol. 145:5-26.

Cherdthong, A., M. Wanapat, P. Kongmun, R. Pilajan, and P. Khejornsart. 2010. Rumen fermentation, Microbial protein synthesis and cellulolytic bacterial population of swamp buffaloes as affected by roughage to concentrate ratio. J. Anim. Vet. Adv. 9:1667-1675.

Choi, Y. J., S. S. Lee, J. Y. Song, N. J. Choi, H. G. Sung, S. G. Yun, and J. K. Ha. 2003. Effects of dietary acidogenicity values on rumen fermentation characteristics and nutrients digestibility. Asian-Aust. J. Anim. Sci. 16:1625-1633.

Denman, S. E., N. Tomkins, and C. S. McSweeney. 2005. Monitoring the effect of bromochloromethane on methanogen populations within the rumen using qPCR. In: $2^{\text {nd }}$ International Symposium on Greenhouse Gases and Animal Agriculture (Ed. C. R. Soliva, J. Takahashi and M. Kreuzer). p. 112 ETH Zurich, Switzerland.

Devendra, C. 1988. General approaches to animal nutrition research and their relevance to fish production in the Asian region. In: Finfish Nutrition Research in Asia (Ed. S. S. DeSilva). Heinemannn Asia Singapore, Singapore, pp. 7-24.

Doto, S. P. and J. X. Liu. 2011. Effects of direct-fed microbials and their combinations with yeast culture on in vitro rumen fermentation characteristics. J. Anim. Feed Sci. 20:259-271.

Galyean, M. 1989. Laboratory procedure in animal nutrition research. Department of Animal and Life Science. New Mexico State University, USA.

Guglielmelli, A., S. Calabrò, M. Cutrignelli, O. Gonzalez, F. Infascelli, R. Tudisco, and V. Piccolo. 2010. In vitro fermentation and methane production of fava and soy beans. EAAP Scientific Series 127:457-460.

Hungate, R. E. 1966. The rumen and its microbes. Academic Press, New York, pp. 53.

Iqbal, M. F., Y. F. Cheng, W. Y. Zhu, and B. Zeshan. 2008. Mitigation of ruminant methane production: current strategies, constraints and future options. World J. Microbiol. Biotechnol. 24:2747-2755.

Jouany, J. P. 2006. Optimizing rumen functions in the close-up transition period and early lactation to drive dry matter intake and energy balance in cows. Anim. Reprod. Sci. 96: 250-264.

Koike, S. and Y. Kobayashi. 2001. Develop and use of competitive PCR assays for the rumen cellulolytic bacteria: Fibrobactor succinogenes, Ruminococcus albus and Ruminococcus flavefaciens. FEMS Microbiol. Lett. 204:361-366.

Lindberg, J. E. 1981. Rumen degradation pattem of dry matter and nitrogenous compounds of some concentrates studied with the nylon bag technique. Swedish J. Agric. Res. 11:171-176.

Lovett, D. K., L. J. Stack, S. Lovell, J. Callan, B. Flynn, and M. Hawkins. 2005. Manipulating enteric methane emissions and animal performance of late-lactation dairy cows through concentrate supplementation at pasture. J. Dairy Sci. 88:28362842.

Lusin, R. and M. Wanapat. 2010. Effect of roughage to concentrate ratio and rice bran oil supplementation on rumen fermentation characteristics using in vitro gas production technique. $14^{\text {th }}$ AAAP Conference at Pingtung University, Taiwan. pp. 353-356.

Lynch, H. A. and S. A. Martin. 2002. Effects of Saccharomyces cerevisiae culture and Saccharomyces cerevisiae live cells on in vitro mixed ruminal microorganism fermentation. J. Dairy Sci. 85:2603-2608.

McGinn, S. M., K. A. Beauchemin, T. Coates, and D. Colombatto. 2004. Methane emissions from beef cattle: Effects of monensin, sunflower oil, enzymes, yeast, and fumaric acid. J. Anim. Sci. 82:3346-3356.

Menke, K. H. and H. Steingass. 1988. Estimation of the energetic feed value obtained from chemical analysis and gas production using rumen fluid. Anim. Res. Dev. 28:7-55.

Moorby, J. M., R. J. Dewhurst, R. T. Evans, and J. L. Danelon. 2006. Effects of dairy cow diet forage proportion on duodenal nutrient supply and urinary purine derivative excretion. J. Dairy Sci. 89:3552-3562.

Mosoni, P., F. Chaucheyras-Durand, C. Béra-Maillet, and E. Forano. 2007. Quantification by real-time PCR of cellulolytic bacteria in the rumen of sheep after supplementation of a forage diet with readily fermentable carbohydrates: effect of a yeast additive. J. Appl. Microbiol. 103:2676-2685.

Moss, A. R., J. P. Jouany, and J. Newbold. 2000. Methane 
production by ruminants: its contribution to global warming. J. Ann. Zootech. 49:231-253.

Oboh, G. 2006. Nutrient enrichment of cassava peels using a mixed culture of Sacchromyces cerevisiae and Lactobacillus spp. solid media fermentation technique. Electron. J. Biotechnol. 9:46-49.

Oboh, G. and A. A. Akindahinsi. 2003. Biochemical changes in cassava products (flour \& gari) subjected to Sacchromyces cerevisiae solid media fermentation. Food Chem. 82:599-602.

Oeztuerk, H. 2009. Effect of live and autoclaved yeast cultures on ruminal fermentation in vitro. J. Anim. Feed Sci. 18:142-150.

Ørskov, E. R. and I. McDonald. 1979. The estimation of protein degradability in the rumen from incubation measurements weighted according to rate of passage. J. Agric. Sci. 92:499503.

Polyorach, S., M. Wanapat, and N. Sornsongnern. 2010. Effect of yeast fermented cassava chip protein (YEFECAP) in concentrate of lactating dairy cows. In: Proceedings of the 14th Animal Science Congress of the Asian-Australasian Association of Animal Production Societies (AAAP), vol. 3, August 23-26, 2010. National Pingtung University of Science and Technology, Pingtung, Taiwan, Republic of China. pp. 304-307.

Polyorach, S., M. Wanapat, and S. Wanapat. 2012. Increasing protein content of cassava (Manihot esculenta, Crantz) using yeast in fermentation. Khon Kaen Agr. J. 40 (Suppl 2):178-182.

Polyorach, S., M. Wanapat, and S. Wanapat. 2013. Enrichment of protein content in cassava (Manihot esculenta Crantz) by supplementing with yeast for use as animal feed. Emirat. J. Food Agric. 25:142-149.

Poungchompu, O., M. Wanapat, C. Wachirapakorn, S. Wanapat, and A. Cherdthong. 2009. Manipulation of ruminal fermentation and methane production by dietary saponins and tannins from mangosteen peel and soapberry fruit. Arch. Anim. Nutr. 63:389-400.

Samuel, M., S. Sagathewan, J. Thomus, and G. Mathen. 1997. An HPLC method for estimation of volatile fatty acids of rumen fluid. Indian J. Anim. Sci. 67:805-807.

SAS. 1998. User's Guide: Statistic, Version 6, 12th ed. SAS Inst. Inc., Cary, NC.

Schoeman, E. A., P. J. De Wet, and W. Burger. 1972. The evaluation of the digestibility of treated proteins. Agroanilnalia 4:35.

Siddons, R. C. and J. Paradine. 1981. Effect of diet on protein degrading activity in sheep rumen. J. Sci. Food Agric. 32:973981.

Slyter, L. L. 1976. Influence of acidosis on rumen function. J. Anim. Sci. 43:910-929.

Steel, R. G. D. and J. H. Torrie. 1980. Principles and Procedures of Statistics. McGraw Hill Book Co, New York, USA.

Sutton, J. D., M. S. Dhanoa, S. V. Morant, J. France, D. J. Napper, and E. Schuller. 2003. Rates of production of acetate, propionate, and butyrate in the rumen of lactating dairy cows given normal and low-roughage diets. J. Dairy Sci. 86:36203633.

Sylvester, J. T., S. K. R. Karnati, Y. Zhongtang, M. Morrison, and J. L. Firkins. 2004. Development of an assay to quantify rumen ciliate protozoal biomass in cows using real-time PCR. J. Nutr. 134:3378-3384.
Tafaj, M., V. Kolaneci, B. Junck, A. Maulbetsch, H. Steingass, and W. Drochner. 2005. Influence of fiber content and concentrate level on chewing activity, ruminal digestion, digesta passage rate and nutrient digestibility in dairy cows in late lactation. Asian-Aust. J. Anim. Sci. 18:1116-1124

Tilley, J. M. A. and R. A. Terry. 1963. A two-stage technique for the in vitro digestion of forage crops. J. Br. Grassland Soc. 18:104-111.

Van Soest, P. J. 1982. Nutritional ecology of the ruminant. O \& B Books Inc, Corvallis.

Van Soest, P. J., J. B. Robertson, and B. A. Lewis. 1991. Methods for dietary fiber, neutral detergent fiber, and nonstarch polysaccharides in relation to animal nutrition. J. Dairy Sci. 74:3583-3597.

Wanapat, M. 2003. Manipulation of cassava cultivation and utilization to improve protein to energy biomass for livestock feeding in the tropics. Asian-Aust. J. Anim. Sci. 16:463-472.

Wanapat, M. and S. Khampa. 2007. Effect of levels of supplementation of concentrate containing high levels of cassava chip on rumen ecology, microbial $\mathrm{N}$ supply and digestibility of nutrients in beef cattle. Asian-Aust. J. Anim. Sci. 20:75-81.

Wanapat, M. and P. Rowlinson. 2007. Nutrition and feeding of swamp buffalo: feed resources and rumen approach. Ital. J. Anim. Sci. 6:67-73.

Wanapat, M. and O. Pimpa. 1999. Effect of ruminal $\mathrm{NH}_{3}-\mathrm{N}$ levels on ruminal fermentation, purine derivatives, digestibility and rice straw intake in swamp buffaloes. Asian-Aust. J. Anim. Sci. 12:904-907.

Wanapat, M., S. Polyorach, K. Boonnop, C. Mapato, and A. Cherdthong. 2009. Effects of treating rice straw with urea or urea and calcium hydroxide upon intake, digestibility, rumen fermentation and milk yield of dairy cows. Livest. Sci. 125:238-243

Wanapat, M., S. Polyorach, V. Chanthakhoun, and N. Sornsongnern. 2011. Yeast-fermented cassava chip protein (YEFECAP) concentrate for lactating dairy cows fed on urealime treated rice straw. Livest. Sci. 139:258-263.

Wora-anu, S., M. Wanapat, C. Wachirapakorn, and N. Nuntaso. 2000. Effects of roughage to concentrate ratio on ruminal ecology and voluntary feed intake in cattle and swamp buffaloes fed on urea- treated rice straw. Asian-Aust. J. Anim. Sci. 13(Suppl.):236-236.

Wright, A. G., A. J. Williams, B. Winder, C. T. Christophersen, S. L. Rodgers, and K. D. Smith. 2004. Molecular diversity of rumen methanogens from sheep in western Australia. Appl. Environ. Microbiol. 70:1263-1270.

Yan, T., R. E. Agnew, F. J. Gordon, and M. G. Porter. 2000. Prediction of methane energy output in dairy and beef cattle offered grass silage based diets. Livest. Prod. Sci. 64:253-263.

$\mathrm{Yu}, \mathrm{Z}$. and M. Morrison. 2004. Improved extraction of PCRquality community DNA from digesta and fecal samples. Bio Techniques 36:808-812.

Zicarelli, F., S. Calabrò, M. I. Cutrignelli, F. Infascelli, R. Tudisco, F. Bovera, and V. Piccolo. 2011. In vitro fermentation characteristics of diets with different forage/concentrate ratios: Comparison of rumen and faecal inocula. J. Sci. Food Agric. 91:1213-1221. 